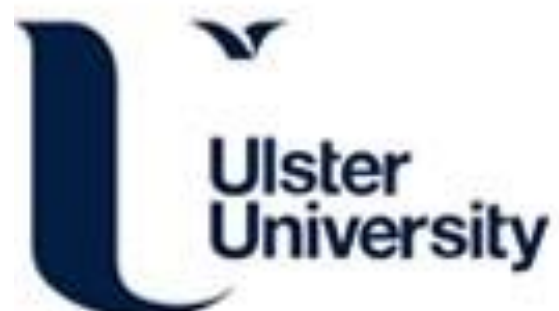

\section{Electrochemical and oxygen reduction properties of pristine and nitrogen-doped few layered graphene nanoflakes (FLGs)}

Soin, N., Roy, SS., Sharma, S., Thundat, T., \& McLaughlin, JAD. (2013). Electrochemical and oxygen reduction properties of pristine and nitrogen-doped few layered graphene nanoflakes (FLGs). Journal of Solid State Electrochemistry, online. https://doi.org/10.1007/s10008-013-2073-8

Link to publication record in Ulster University Research Portal

Published in:

Journal of Solid State Electrochemistry

Publication Status:

Published (in print/issue): 01/01/2013

DOI:

10.1007/s10008-013-2073-8

\section{Document Version}

Publisher's PDF, also known as Version of record

\section{General rights}

Copyright for the publications made accessible via Ulster University's Research Portal is retained by the author(s) and / or other copyright owners and it is a condition of accessing these publications that users recognise and abide by the legal requirements associated with these rights.

\section{Take down policy}

The Research Portal is Ulster University's institutional repository that provides access to Ulster's research outputs. Every effort has been made to ensure that content in the Research Portal does not infringe any person's rights, or applicable UK laws. If you discover content in the Research Portal that you believe breaches copyright or violates any law, please contact pure-support@ulster.ac.uk. 


\title{
Electrochemical and oxygen reduction properties of pristine and nitrogen-doped few layered graphene nanoflakes (FLGs)
}

\author{
Navneet Soin • Susanta Sinha Roy • Surbhi Sharma • \\ Thomas Thundat • James A. McLaughlin
}

Received: 14 January 2013 / Revised: 20 March 2013 / Accepted: 22 March 2013

(C) Springer-Verlag Berlin Heidelberg 2013

\begin{abstract}
Vertically aligned few layered graphene (FLGs) nanoflakes were synthesized by microwave plasma deposition for various time durations ranging from 30 to $600 \mathrm{~s}$ to yield graphene films of varying morphology, microstructure and areal/edge density. Their intrinsic electrochemical properties were explored using $\mathrm{Fe}(\mathrm{CN})_{6}{ }^{3-/ 4-}$ and $\mathrm{Ru}\left(\mathrm{NH}_{3}\right)_{6}{ }^{3+/ 2+}$ redox species. All the FLG electrodes demonstrate fast electron transfer kinetics with near ideal $\Delta E \mathrm{p}$ values of 60-65 mV. Using a relationship between electron transfer rate and edge plane density, an estimation of the edge plane density was carried out which revealed a moderation of edge plane density with increase in growth time. The pristine FLGs also possess excellent electrocatalytic activity towards oxygen reduction reaction (ORR) in alkaline solutions. This
\end{abstract}

Electronic supplementary material The online version of this article (doi:10.1007/s10008-013-2073-8) contains supplementary material, which is available to authorized users.

N. Soin · J. A. McLaughlin

Nanotechnology and Integrated Bioengineering Centre (NIBEC), University of Ulster at Jordanstown, Shore Road,

Newtownabbey BT37 0QB, UK

\section{S. S. Roy}

Department of Physics, School of Natural Sciences, Shiv Nadar

University, Gautam Budh Nagar 203207, Uttar Pradesh, India

S. Sharma

School of Chemical Engineering, University of Birmingham,

Edgbaston, Birmingham B15 2TT, UK

T. Thundat

Chemical and Materials Engineering Department,

University of Alberta, Edmonton, AB T6G 2V4, Canada

Present Address:

N. Soin $(\square)$

Institute of Renewable Energy and Environmental Technologies (IREET), Knowledge Centre for Materials Chemistry (KCMC), University of Bolton, Bolton BL3 5AB, UK

e-mail: soin-n@email.ulster.ac.uk
ORR activity can be further enhanced by exposing the pristine FLGs to nitrogen electron cyclotron resonance plasma. The metal free N-doped FLGs exhibit much higher electrocatalytic activity towards ORR than pristine FLGs with higher durability and selectivity than Pt-based catalysts. The excellent electrochemical performance of N-doped FLGs is explained in terms of enhanced edge plane exposure, high content of pyridinic nitrogen and an increase in the electronic density of states.

Keywords Graphene - Few layered graphene nanoflakes . Nitrogen doping · Electrochemical properties · Oxygen reduction reaction

\section{Introduction}

Carbon-based electrodes diversify across a breadth of applications including electroanalysis, biosensing, electrocatalysis, fuel cell electrodes and energy storage elements among others [1-3]. The basic building block of graphene and structural polymorphism of carbon is responsible for a wide variety of carbon-based electrodes such as highly oriented pyrolytic graphite (HOPG), boron-doped diamond, glassy carbon (GCE) and nanotubes (CNTs). Also, the high chemical stability along with rich surface chemistry allows a large number of functional groups to be attached for further applications [1-4]. These electrodes offer the advantages of wide potential window, electrocatalytic activity for redox probes, intrinsic inert electrochemistry, good electrical conductivity and comparatively low cost as compared to noble metal( $\mathrm{Au}, \mathrm{Pt}$ )-based electrodes $[1,4]$.

The inherent electroactivity of the carbon-based electrodes depends upon on the microstructure, surface chemistry and electronic structure and most importantly on the amount of the highly reactive edge planes in the electrode [1, 2, 4-6]. The edge planes exhibit fast electron transfer as compared to the basal planes which are nearly dormant and 
exhibit poor electron transfer rates [1, 2, 4-6]. While HOPG and GCE are ideal materials for electrochemical studies, they suffer from the problems of high cost and the need of careful polishing to expose the active edge planes. Over the past decade or so, CNTs have been widely shown to exhibit high electroactivity and fast electron transfer rates $[1,3]$. However, recent reports have suggested that the inherent activity of CNTs is due to the presence of "irremovable" metal nanoparticles encapsulated during the growth of CNTs, potentially leading to misinterpretation of results and affecting the repeatability and long-term stability [3-8]. Hence, it is of utmost importance to look at new catalyst free carbon nanostructures which not only provide extensive edge plane structure and have the ability to catalyse important electrochemical reactions. We have recently reported on the catalyst free growth of few layered graphene (FLGs) nanoflakes; which are essentially vertically stacked two-dimensional graphene sheets growing from the underlying substrate terminating into open graphitic edge planes [9-12]. ${ }^{1}$ The high degree of edge planes provides a large number of active sites for fast heterogeneous electron transfer along the graphene backbone [9-12].

Relating the amount of edge plane or defect sites on carbon nanomaterials to their electrochemical properties is of fundamental importance for electrochemical applications $[1,2]$. The role of edge planes for fast electron transfer is now well established, and electrochemical protocols have been devised to ascertain the density of edge planes/defect sites $[2,5,6,13]$. The usual techniques of high resolution electron microscopy and Raman spectroscopy are time consuming and not readily available [4-6]. A capacitance-based measurement has been proposed by Pumera et al. to compute the edge sites [13]. More recently, Banks et al. have proposed a method which relies on the estimation of heterogeneous electron transfer rate and its relationship with the defect density, which is then compared to a simulation of electron transfer rate on a purely edge plane electrode $[5,6]$. Using this method, an estimation of the active edge sites of the FLGs have been performed as a function of growth time and is co-related with their microstructure.

Heteroatom doping of carbon nanomaterials, especially nitrogen doping, has been shown to modulate the electronic, structural and chemical properties for a variety of applications including oxygen reduction reaction (ORR) [14-16].

\footnotetext{
${ }^{1}$ There is a lot of discrepancy and disparity in the literature regarding the nomenclature used for carbon nanostructures like these such as few-layered graphene nanoflakes, multi-layered graphene petals and carbon nanowalls. Strictly speaking, graphene is a single sheet of $\mathrm{sp}^{2}$ hybridised carbon atoms; hence, the use of term few-layered graphene can be considered as slightly controversial. In the past, we have reported on the synthesis, growth mechanism and applications of these structures wherein they have been addressed as few-layered graphene nanoflakes. So, in order to maintain parity, we continue to call it as few-layered graphene nanoflakes.
}

The traditional Pt-based catalyst not only suffers from high susceptibility towards methanol crossover effects but also high cost [14-16]. Hence, extensive research is being undertaken to discover new metal-free catalysts which can offer high selectivity for ORR and can sustain methanol crossover effects. Recent report by Dai et al. showed high electrocatalytic activity as well as long-term stability of nitrogen-doped CNTs for ORR [14]. Excellent ORR has been reported on $\mathrm{N}$-doped graphene as well [16-18, 21]. It has been proposed that the activity of nitrogenated carbon nanomaterials towards ORR stems from (1) production of partial positive charge on bonded nitrogen/oxygen bonded carbon atom, (2) nitrogen moieties acting as active sites [14-21]. Also, the high amount of localised electronic density of states (DOS) in nitrogen-doped graphene in conjunction with higher defect density may help in catalyzing the reduction of oxygen [14-18].

In the present work, we report on the basic electrochemical properties of FLGs including electron transfer kinetics and an estimation of electro-active edge sites using cyclic voltammetry response to redox species such as $\mathrm{Fe}(\mathrm{CN})_{6}^{3-/ 4-}$ and $\mathrm{Ru}\left(\mathrm{NH}_{3}\right)_{6}{ }^{3+/ 2+}$. The influence of exposure of edge planes on the electron transfer rate has been studied in detail. Low pressure nitrogen electron cyclotron resonance (ECR) plasma treatment was carried out to functionalize the FLG surface via doping of various nitrogen moieties. The influence of enhanced electronic density of states and presence of nitrogen moieties is also discussed in relation to reduction of molecular oxygen on FLGs.

\section{Experimental}

The synthesis of FLGs was carried out in a SEKI microwave plasma enhanced chemical vapour deposition system, equipped with a $1.5 \mathrm{~kW}, 2.45 \mathrm{GHz}$ microwave source. The substrates used were bare in type heavily doped Si wafers (resistivity $<0.005 \Omega \mathrm{cm}$ ). Prior to growth, the substrates are pre-treated with $\mathrm{N}_{2}$ plasma at $650 \mathrm{~W}$ at 40 Torr, while the substrate temperature is maintained at $900{ }^{\circ} \mathrm{C}$. Synthesis was then carried out using $\mathrm{CH}_{4} / \mathrm{N}_{2}$ (gas flow ratio 1:4) plasma at $800 \mathrm{~W}$ for the desired duration. For further nitrogen doping, in-situ ECR plasma treatment of samples was carried out at a working pressure of $\sim 0.025 \mathrm{~Pa}(1.8 \times$ $10^{-4}$ Torr), while the microwave power was maintained at $150 \mathrm{~W}$ for a various time durations of 300 and $600 \mathrm{~s}$ [9-12]. The conditions used are similar to the one used in our previous works [9-12].

Scanning electron microscopy (SEM) was performed on a FEI Quanta 3D operating at $30 \mathrm{kV}$ while, transmission electron microscopy (TEM) analysis was performed on a JEOL JEM-4000EX operating at $100 \mathrm{kV}$, for which samples were scraped off the surface and deposited on a 300 mesh 
holey carbon grids. Atomic force microscopy (AFM) images were obtained using a Veeco Nanoman DI 3100 AFM system. X-ray photoelectron spectroscopy (XPS) analysis was performed on a Kratos Axis Ultra DLD spectrometer using a monochromatic Al $K \alpha$ X-rays $(h v=1,486.6 \mathrm{eV})$, operating at $15 \mathrm{kV}$ and $10 \mathrm{~mA}$. Raman spectroscopy was performed using an ISA LabRam system equipped with a $632.8 \mathrm{~nm} \mathrm{He}-\mathrm{Ne}$ laser. Electrochemical analysis were carried out using the customised home-made electrochemical cell in a three electrode setup using $\mathrm{Ag} / \mathrm{AgCl}$, Pt wire and FLGs as reference, counter and working electrode, respectively. The electrochemical cell allowed a constant $3 \mathrm{~mm}$ working area using an O-ring with the FLG samples being held in contact with the copper plate using a sticky copper tape to maximise the contact area. The electrolytes used was $1 \mathrm{mM} \mathrm{Ru}\left(\mathrm{NH}_{3}\right)_{6} \mathrm{Cl}_{3}$ in $1 \mathrm{M} \mathrm{KCl}$ and $5 \mathrm{mM} \mathrm{K}_{4} \mathrm{Fe}(\mathrm{CN})_{6}$ in $1 \mathrm{M} \mathrm{KCl}$. Further experiments for ORR were carried out using $0.1 \mathrm{M} \mathrm{KOH}$ electrolyte, which was saturated with $\mathrm{N}_{2}$ or $\mathrm{O}_{2}$ for at least $15 \mathrm{~min}$ prior to running the scans and kept under $\mathrm{N}_{2}$ or $\mathrm{O}_{2}$ ambient during the scans. The FLG samples were used as "as-prepared" after the deposition (for pristine samples) and nitrogen doping (for N-doped samples). As the electrode preparation did not involve any scraping off the sample from the $\mathrm{Si}$-substrate; hence, there was no need for the use of any Nafion for ink-preparation.

\section{Results and discussion}

The overall morphology and structure of the obtained FLGs were characterised by electron microscopy and AFM analysis. The SEM image of FLGs (grown for $60 \mathrm{~s}$ ) is shown in Fig. 1(a) whereas the edge structure of flakes is shown in the TEM image (Fig. 1(b)), clearly demonstrating the few-layered graphene nature of the structures. The three-dimensional AFM images of FLGs grown at various time durations are shown in Fig. 2(a-c). These growth times have been chosen as they represent three different microstructures in terms of their crystallite size, edge density and distribution (see supporting information S1) [9-12]. The films consist of robust and sharp flake-like structures with randomly interwoven sharp edges with open spaces between them. It has been previously reported that with an increase in the growth time, the degree of interlinking increases along with an overall increase in the height of the flakes [9-12]. Also, the number of effective FLG edge sites reduces due to amalgamation of different flake edges accompanied by an increase in the thickness of the flake edges [9]. In the Raman spectra shown in Fig. 2(d), the overall change in the microstructure can be clearly observed. For samples grown for $30 \mathrm{~s}$, the samples are characterised by a high defect peak ( $\mathrm{D}$ band) due to a higher number density of FLGs. With an increase in the growth time $(60 \mathrm{~s})$, the intensity of the $G$ band increases slightly due to a consolidation of FLGs reducing the number of edge plane structures. The two-dimensional nature of FLGs grown for 30 and $60 \mathrm{~s}$ is evident by the high intensity $\left(\mathrm{I}_{\mathrm{G}} / \mathrm{I}_{\mathrm{G}}>1\right)$ and narrow full width at half maximum of $\sim 43 \mathrm{~cm}^{-1}$ for the $\mathrm{G}^{\prime}$ peak [9-12, 22]. For the samples grown for $600 \mathrm{~s}$, while, there is a significant reduction in the $\mathrm{I}_{\mathrm{G}} / \mathrm{I}_{\mathrm{G}}$ ratio; however, no splitting of the $\mathrm{G}^{\prime}$ band was observed which occurs for three-dimensional graphitic structures thereby confirming the two-dimensional nature of FLGs across the deposition regime [9-12, 22]. For the nitrogen doped FLGs, the enhancement in the defects can be clearly observed (see supporting information, S1). The heteroatom doping in graphene has been shown to produce pentagonal defects in the honeycomb lattice structure leading them to distort severely and creating enhanced defect sites. While the pristine FLGs show highly parallel planes with a regular $\mathrm{d}$ spacing, the N-doped samples exhibit large fluctuations in $\mathrm{d}$ spacing and appear severely distorted as shown in Fig. S2 (supporting information). Similar signatures of increase in the disorder can be observed in the Raman spectroscopy analysis of the pristine and N-doped FLGs (see supporting information S2). This increase in the disorder has implications for the electrochemical properties of the N-doped FLGs as discussed later on.
Fig. 1 a High magnification SEM image and $\mathbf{b}$ high resolution TEM image showing few layered nature of FLGs (arrow shows the graphitic edges present)

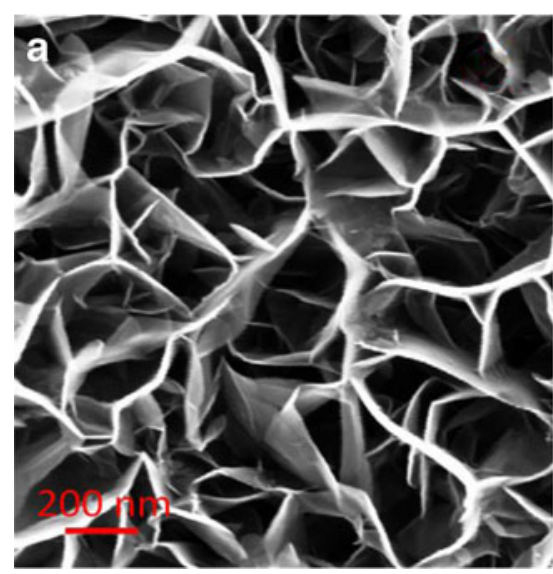

b

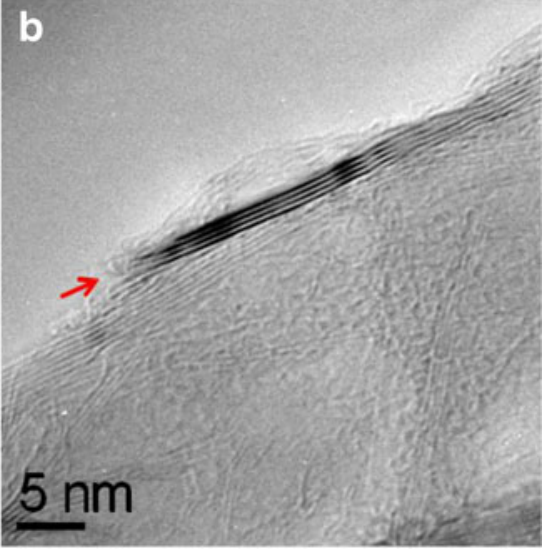


Fig. 2 AFM images of FLGs grown for a $30, \mathbf{b} 60$ and $\mathbf{c}$ $600 \mathrm{~s}$ showing the effective change in the morphology and structure of flakes. d Corresponding Raman spectra shows the change in the microstructure of FLGs
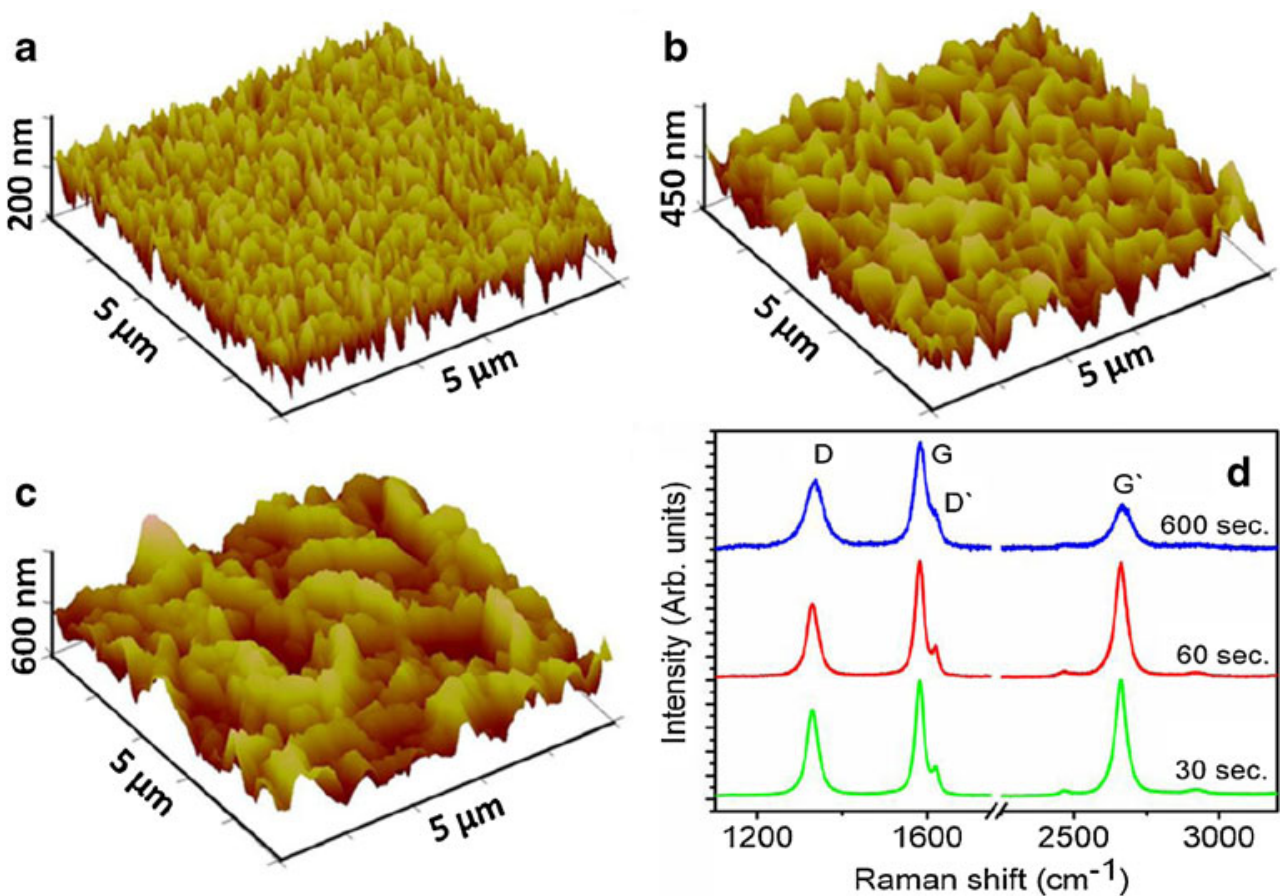

\section{Electrochemical properties of FLGs}

The vertically aligned highly graphitized edge plane structure of the FLGs presents an ideal system to study the role of edge planes in electrochemical reactions. Also, the absence of any metal catalyst ensures that the observed electrochemical response is the true behaviour of the electrode $[1,11,23$, 24]. Figure 3(a-c) shows the electrochemical response of FLGs to $5 \mathrm{mM} \mathrm{K}_{4} \mathrm{Fe}(\mathrm{CN})_{6}$ in $1 \mathrm{M} \mathrm{KCl}$ electrolyte. It should be mentioned here that $\mathrm{K}_{4} \mathrm{Fe}(\mathrm{CN})_{6}$ has been chosen, as it displays a single electron transfer reaction which is well understood for a large variety of $\mathrm{sp}^{2}$ and $\mathrm{sp}^{3}$ carbon systems $[1,11,23-25]$. All the three samples show well-defined peaks in the forward and reverse scans due to the oxidation and reduction of the $\mathrm{Fe}(\mathrm{CN})_{6}{ }^{3-/ 4-}$ redox couple. The reversible redox reaction on FLGs is further confirmed by the observed linear anodic $\left(I_{\mathrm{pa}}\right)$ and cathodic $\left(I_{\mathrm{pc}}\right)$ peak currents vs. $v^{1 / 2}$ plots as shown in Fig. 3(d) [11, 23-25]. The data is indicative of the nature of the reaction, which is limited by semi-infinite linear diffusion of the reactants to the electrode surface [23-26]. However, the observed current density $\left(I_{\mathrm{p}}\right)$ reduces with the increase in the growth time. In order to explain this, let us consider the Randles-Sevcik equation:

$I_{p}=2.687 \times 10^{5} A C n^{\frac{3}{2}}(D v)^{\frac{1}{2}}$

Where $I_{\mathrm{p}}$ is the peak current (A), $A$ is the electroactive area (square centimetre), $C$ is the concentration of electroactive species (cubic metres per mole), $n$ is the number of electrons taking part in the redox, $D$ is the diffusion coefficient (centimeter-squared per second) and $v$ is the scan rate $\left(\mathrm{Vs}^{-1}\right)$ [26-28]. For Eq. 1, it can be clearly observed that for a particular electrochemical redox system, the peak current is directly related to the scan rate and the effective electrode area. Since all the samples were analyzed with the same redox probe and at same scan rates, the only variable that can contribute towards the change in the redox current is the effective electrode area $(A)$. This area is entirely different from the geometrical area and is related to the amount of electroactive sites on the sample [26-29]. Indeed, it has been shown that for $\mathrm{sp}^{2}$ carbon nanomaterials, the relative proportion of edge planes exposed to the electrolyte solution is directly proportional to the rate of reaction and the observed current $[1,2,30,31]$. Using the above equation, the effective area of the electrodes was calculated to be $0.336,0.289$ and $0.206 \mathrm{~cm}^{2}$ for samples grown for 30,60 and $600 \mathrm{~s}$, respectively. In the AFM images of samples shown in Fig. 2(a-c), it can be clearly observed that with the increase in the growth time, the degree of interlinking between the flakes increases thereby reducing the actual number of exposed edges (active sites). Since the decrease in the edge sites is directly proportional to the rate of the electron transfer, the reduction in the observed current density can be clearly accounted for.

The most important parameter for any electrode is its electron transfer kinetics and the peak to peak separation values $\left(\Delta E_{\mathrm{p}}\right)$. The values of $\Delta E_{\mathrm{p}}$ are strongly dependent on the electron transfer rate, i.e. the reactivity of the electrode towards the electrolyte $[1-6,11]$. For all the samples, welldefined peaks in the forward and reverse scans were observed corresponding to the oxidation and reduction of $\mathrm{Fe}(\mathrm{CN})_{6}^{3-/ 4-}$ 
Fig. $3 \mathrm{CV}$ response of pristine FLGs grown at a 30, b 60 and $\mathbf{c}$ 600 s. d Plot of peak current values vs. square root of the scan rate in $5 \mathrm{mM} \mathrm{K}_{4} \mathrm{Fe}(\mathrm{CN})_{6}$ in $1 \mathrm{M} \mathrm{KCl}$
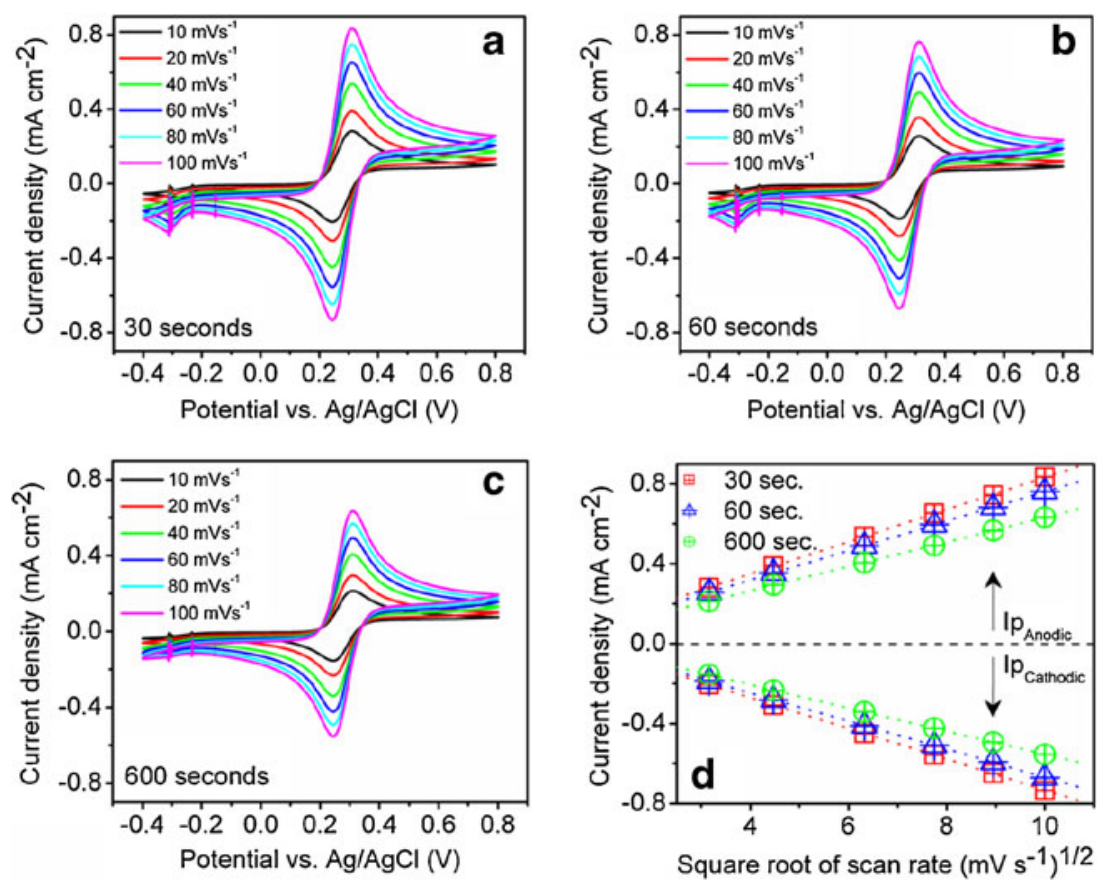

species. The observed electrochemical response showed nearly perfect reversible Nerstian behaviour with a peak to peak separation $(\Delta E \mathrm{p})$ of nearly $60 \mathrm{mV}[11,23]$. In fact, the values of $\Delta E_{\mathrm{p}}$ were $60 \pm 2 \mathrm{mV}, 63 \pm 2 \mathrm{mV}$ and $65 \pm 3 \mathrm{mV}$ for samples grown for 30, 60 and $600 \mathrm{~s}$, respectively. The values obtained here are obtained on pristine FLGs without the need for any surface activation procedures such as polishing etc. The values of $\Delta E_{\mathrm{p}}$ are very close to the ideal peak separation for single electron transfer reaction of $59 \mathrm{mV}$ [1, 2, 11, 23]. More importantly, the electron transfer rate and the associated $\Delta E_{\mathrm{p}}$ can be fully attributed to the properties of the FLGs itself. Nicholson developed and published a simple method to evaluate the standard rate constant for the electron transfer from the peak potential separation and the scan rate of a quasireversible system. In the original work by Nicholson, the cyclic voltammetry response profile for a quasi-reversible electron transfer reaction was subjected to quantitative mathematical analysis [32]. The net shape of the voltammetry curves have been shown to depend on a kinetic parameter $\psi$ which is given by the expression shown below (Eq. 2). The method provides an extremely rapid and simple way to evaluate electrode kinetics. Using the peak to peak separation, $\Delta E_{\mathrm{p}}$, the apparent electrochemical rate constant $\left(k_{0}\right)$ can be determined using the Nicholson's method given by the following equation:

$k_{0}=\Psi\left[\frac{\pi D_{\mathrm{o}} n F v}{R T}\right]^{\frac{1}{2}}\left(\frac{D_{\mathrm{r}}}{D_{\mathrm{o}}}\right)^{\frac{\alpha}{2}}$

where $\Psi$ is the kinetic parameter, $k_{0}$ is the rate constant, $D_{0}$ is the diffusion constant of $\mathrm{Fe}(\mathrm{CN})_{6}{ }^{3-/ 4-}\left(7.63 \times 10^{-6} \mathrm{~cm}^{2} \mathrm{~s}^{-1}\right.$, $1 \mathrm{M} \mathrm{KCl}), n$ is the number of electrons transferred, $F$ is the
Faraday's constant, $v$ is the scan rate, $R$ is the universal gas constant, $T$ is the temperature and is the transfer coefficient (0.5) [5, 6, 30-32]. For all the samples, the peak to peak separation, $\Delta E_{\mathrm{p}}$, was large enough $(\geq 59 \mathrm{mV})$ to find out the kinetic parameter, $\Psi$ using the Nicholson method [30, 32]. The value of standard electrochemical rate constant $\left(k_{0}\right)$ was found to be $6.1 \times 10^{-2} \mathrm{~cm} \mathrm{~s}^{-1}, 2.13 \times 10^{-2} \mathrm{~cm} \mathrm{~s}^{-1}$ and $1.5 \times$ $10^{-2} \mathrm{~cm} \mathrm{~s}^{-1}$ for samples grown for 30,60 and $600 \mathrm{~s}$, respectively, and are shown in Table 1. It should be noted here that these values should not be considered as absolute values as no correction was made for $i R$ effects or double layer effects. Also, the character of Nicholson's working curve is such that even a change of $\pm 1 \mathrm{mV}$ has a profound effect on the value of $\Psi$ and consequently on the value of calculated electrochemical rate constant [32]. In our case, where the FLG samples have $\Delta E_{\mathrm{p}}$ values which are so close to each other, the electrochemical rate constants should be treated as apparent and indicative rate constants only. The slightly higher value of standard electrochemical rate constant $\left(k_{0}\right)$ for samples grown at $30 \mathrm{~s}$ is in agreement with the nearly ideal peak to peak separation observed for these electrodes. These results are in agreement with those shown by Tang et al. in their study of electrochemical properties of reduced graphene sheets [25]. The RandlesSevcik equation was derived with a key assumption that the electrode surface is one-dimensional, i.e. the surface is flat without any protrusions or large topographical features [26-28]. Hence, it has been argued that the Randles-Sevcik equation can only be used for the calculation of electroactive surface area when the rigid electrode has a definite surface roughness rather than having a porous structure. In our case, the FLG nanoflakes are quite randomly distributed on the 
Table 1 The values of electrochemical rate constant and edge plane exposure for $\mathrm{Fe}(\mathrm{CN})_{6}{ }^{3-/ 4-}$ and $\mathrm{Ru}\left(\mathrm{NH}_{3}\right)_{6}{ }^{2+/ 3+}$ redox probes for samples grown for 30,60 and $600 \mathrm{~s}$

\begin{tabular}{|c|c|c|c|c|c|}
\hline \multirow{2}{*}{$\begin{array}{l}\text { Sample } \\
\text { Growth time (sec) }\end{array}$} & \multicolumn{2}{|c|}{$\mathrm{Fe}(\mathrm{CN})_{6}^{3-/ 4-}$} & \multicolumn{3}{|c|}{$\mathrm{Ru}\left(\mathrm{NH}_{3}\right)_{6}{ }^{2+/ 3+}$} \\
\hline & $\Delta E_{\mathrm{p}}(\mathrm{mV})$ & $k_{o b s}^{0}\left(\mathrm{~cm} \mathrm{~s}^{-1}\right)$ & $\Delta E_{\mathrm{p}}(\mathrm{mV})$ & $k_{o b s}^{0}\left(\mathrm{~cm} \mathrm{~s}^{-1}\right)$ & $\theta_{\text {edge }}(\%)$ \\
\hline 30 & 60 & $6.1 \times 10^{-2}$ & 64 & $1.72 \times 10^{-2}$ & 4.25 \\
\hline 60 & 63 & $2.1 \times 10^{-2}$ & 66 & $1.1 \times 10^{-2}$ & 2.75 \\
\hline 600 & 65 & $1.5 \times 10^{-2}$ & 71 & $5.6 \times 10^{-3}$ & 1.45 \\
\hline
\end{tabular}

surface, presenting with a nest-like structure representative of a more porous structure. In a structure like this, when soaked in the electrolyte solution, the diffusion occurs through the pores and the channels of the material. From analysis of the AFM images (Fig. 2) of the films grown for various time durations, the distance between the flakes varies from approximately 50-100 (for $30 \mathrm{~s}$ growth time) to 500-1,000 nm (for $600 \mathrm{~s}$ growth time). Now in order for the Randles-Sevcik equation to be valid, the size of the diffusion layer formed (at a particular scan rate) in the average of each ensemble of nanoflakes should be much higher than the average spacing between them $[33,34]$. To calculate this, we need to consider the size of the diffusion layer $\delta$ surrounding the nanoflake ensemble using the equation $\delta=\left(2 \mathrm{D} \times(\Delta \mathrm{E} / \mathrm{v})^{1 / 2}\right)$, where $\mathrm{D}$ is diffusion constant for the ferrocouple redox probe $\left(7.63 \times 10^{-6}\right.$ $\left.\mathrm{cm}^{2} \mathrm{~s}^{-1}, 1 \mathrm{M} \mathrm{KCl}\right), \Delta \mathrm{E}$ is the potential width of the voltammograms, and $v$ is the scan rate. For scan rates of $100 \mathrm{mVs}^{-1}$, the size of the diffusion layer created in the average of each isolated FLG is approximately $50 \mu \mathrm{m}$. Hence, it can be clearly observed that the distance between the FLG flakes $(100-1,000 \mathrm{~nm})$ is much smaller than the size of the diffusion layer $(50 \mu \mathrm{m})$. This is suggestive of the fact that the individual diffusion layers of the FLGs overlap quite heavily leading to the depletion of spaces between the neighbouring FLGs and significantly increasing the planar diffusion. The electrode surface thus obtained is analogous to a category four surface according to the classification developed by Compton and Davies [34]. The mass transport in the case of category four can be described by planar diffusion, so the mass transport can then be reduced to a one-dimensional problem. It is known that the edge plane shows much faster electron transfer kinetics as compared to the basal plane for nearly all the redox couples $[1,2,23]$. An electrode having large amount of edge plane sites would show a nearly reversible behaviour, whereas for a basal plane dominated electrode will show irreversible behaviour. This huge difference in the properties of the edge and basal plane electrodes arises from the large difference in the DOS near the Fermi level. The basal planes usually have a low DOS at Fermi level, and thus have a smaller probability that an electron with the correct energy is available for electron transfer to occur through the redox probe $[1,2,11,23]$. At the edge planes, however, due to a large defect density, a much higher DOS is available due to the production of localised edge states between the conduction and the valence band [1, 11, 23]. Thus, in the case of FLGs where the structure is dominated by a large number of edge planes, an enhanced electron transfer is observed. While the presence of defect sites on the edges of FLGs can significantly enhance the localised DOS and consequently the electron transfer rate, the types of defects itself can influence the edge states [1, $11,23,35]$. These edge states in conjunction with the surface adsorbates (Oxygen in this case) can change the DOS near the Fermi level [22]. In the case of $\mathrm{Fe}(\mathrm{CN})_{6}{ }^{3-/ 4-}$ probe, the presence of oxygen has been shown to adversely affect the electron transfer rate $[23,36]$. For pristine FLGs, XPS analysis revealed a small quantity of adsorbed oxygen on the surface (Table 2). This presence of oxygen on the surface in conjunction with the type of edge states (armchair or zigzag) may be responsible for the slightly higher than the ideal electron transfer rate observed for FLG samples [11, 23, 36]. The inherent two-dimensional structure of the FLGs, consisting of multiple graphene sheets provides high electron mobility for electrons to be transferred from the electrode surface through the underlying $\mathrm{Si}$ substrate, thereby demonstrating good electrical contact between the FLGs and underlying Si. Thus, from the cyclic voltammetry results, it can be clearly concluded that a catalyst free system like FLG with a high degree of active graphitic edge planes demonstrates fast electron transfer kinetics and provides an excellent platform to perform electrochemical studies.

As compared to the $\mathrm{Fe}(\mathrm{CN})_{6}{ }^{3-/ 4-}$ probe, the $\mathrm{Ru}\left(\mathrm{NH}_{3}\right)_{6}{ }^{3+/ 2+}$ is relatively insensitive to the surface microstructure and
Table 2 XPS quantification data for pristine and ECR N-doped FLGs

\begin{tabular}{llll}
\hline Element (atomic \%) & Pristine FLGs & $300 \mathrm{sec}$. ECR N-doped FLGs & $600 \mathrm{sec}$. ECR N-doped FLGs \\
\hline Carbon & $96.60 \pm 0.92$ & $74.81 \pm 0.25$ & $32.41 \pm 2.64$ \\
Oxygen & $3.40 \pm 0.91$ & $17.95 \pm 0.28$ & $50.29 \pm 2.23$ \\
Nitrogen & - & $7.24 \pm 0.13$ & $17.30 \pm 0.93$ \\
\hline
\end{tabular}


Fig. $4 \mathrm{CV}$ response of pristine FLGs grown at a 30, b 60 and $\mathbf{c}$ 600 s. d Plot of peak current values vs. square root of the scan rate in $1 \mathrm{mM} \mathrm{Ru}\left(\mathrm{NH}_{3}\right)_{6} \mathrm{Cl}_{3}$ in $1 \mathrm{M} \mathrm{KCl}$
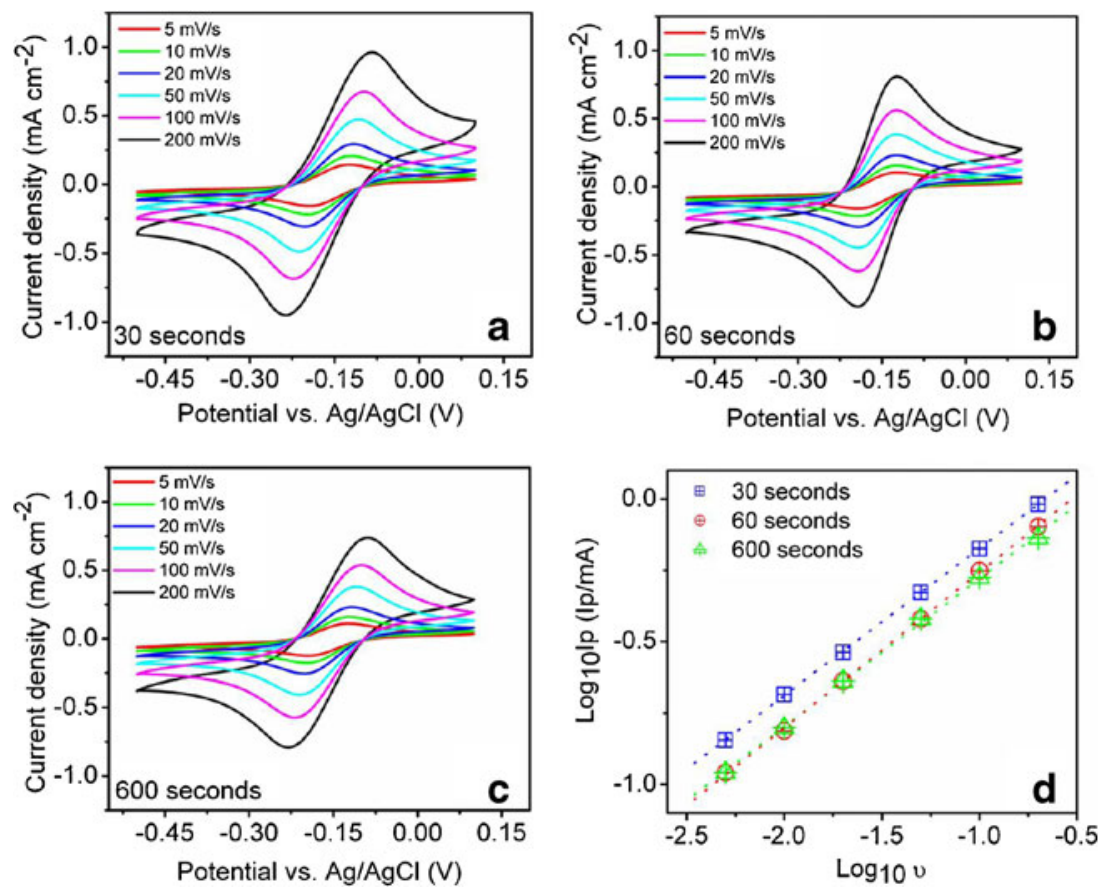

surface oxides for carbon electrodes [1,25]. The only parameter affecting the reaction rate is the electronic DOS near the Fermi level and hence would be unaffected by the adsorbates such as oxides on the surface $[1,5,25]$. The electrochemical response of FLGs at different growth times in $1 \mathrm{mM}$ $\mathrm{Ru}\left(\mathrm{NH}_{3}\right)_{6} \mathrm{Cl}_{3} / 1 \mathrm{M} \mathrm{KCl}$ is shown in Fig. 4(a-c). The electrochemical current response in the case of samples grown for $30 \mathrm{~s}$ was the highest followed by 60 and $600 \mathrm{~s}$, respectively. Also, the value of slope of the linear fittings of peak current vs. the scan rate has values of nearly 0.5 , which is indicative that the reaction proceeds through semi-infinite linear diffusion (Fig. 4(d)) [5, 6]. For all the samples, the peak to peak separation, $\Delta E_{\mathrm{p}}$, was large enough ( $\geq 59 \mathrm{mV}$ ) to find out the kinetic parameter, $\Psi$ using the Nicholson method $\left(D_{\mathrm{o}}\right.$ for $\left.\mathrm{Ru}\left(\mathrm{NH}_{3}\right)_{6} \mathrm{Cl}_{3}=7.63 \times 10^{-6} \mathrm{~cm}^{2} \mathrm{~s}^{-1}\right)[30,32]$. Using the peak to peak separation values in conjunction with Nicholson's method described by Eq. (3), the electrochemical rate constants $\left(k_{\text {obs }}^{0}\right)$ have been calculated and tabulated in Table 1. It should again be mentioned here that the electrochemical rate constants shown in Table 1 are apparent rate constants due to the reasons already discussed above. These values are an order of magnitude higher than those observed on GCE surfaces and are commensurate with those observed by Tang et al. [24].

As mentioned earlier, the quantification of edge sites for electrodes is fundamentally important to understand their electrochemical behaviour. Banks et al. have recently proposed an approach which allows the quantification of the edge plane sites using cyclic voltammetry analysis $[5,6]$. The method allows the determination of a quantitative relationship between electron transfer rate and density of defects viz. edge plane sites for the electrode material. In order to find out the quantity of edge sites, the standard electron transfer rate needs to be calculated which is then compared to the simulated value on an electrode which consists of edge plane sites only. Considering Eq. 2 and assuming the diffusion coefficients for oxidized and reduced species are the same, Eq. 2 is reduced to:

$k_{0}=\Psi\left[\frac{\pi D_{\mathrm{o}} n F v}{R T}\right]^{\frac{1}{2}}$

It has been shown that the observed electron transfer rate, $k_{\mathrm{obs}}^{0}$ on HOPG electrodes has contributions from both the basal plane $\left(k_{\text {basal }}^{0}\right)$ and the edge plane $\left(k_{\text {edge }}^{0}\right)[5,6]$. Thus, the observed electron transfer rate can be written as:

$k_{\text {obs }}^{0}=k_{\text {edge }}^{0}\left(\theta_{\text {edge }}\right)+k_{\text {basal }}^{0}\left(1-\theta_{\text {edge }}\right)$

Where $\theta_{\text {edge }}$ represents the effective number of edge plane sites. Since the electrochemical reactions over edge planes are much faster than those occurring over basal plane $\left(k_{\text {edge }}^{0} \gg k_{\text {basal }}^{0}\right)$, the Eq. (4) is reduced to [5, 6]:

$k_{\text {obs }}^{0}=k_{\text {edge }}^{0}\left(\theta_{\text {edge }}\right)$

In order to quantify the number of edge sites $\left(\theta_{\text {edge }}\right)$ contributing to the effective electron transfer reaction, the value of, $k_{\mathrm{obs}}^{0}$ can be obtained from using the cyclic voltammetry scans in conjunction with Eq. (5). The electron transfer rate for edge plane $\left(k_{\text {edge }}^{0}\right)$ of the $\left[\mathrm{Ru}\left(\mathrm{NH}_{3}\right)_{6}\right]^{3+} /\left[\mathrm{Ru}\left(\mathrm{NH}_{3}\right)_{6}\right]^{2+}$ system has been determined previously using a commercial simulation 
package providing a value of $0.4 \mathrm{~cm} \mathrm{~s}^{-1}[5,6]$. Using the combination of these two values in Eq. 5, the values of $\theta_{\text {edge }}$ (active sites) has been calculated and shown in Table 1. As expected, the values of $\theta_{\text {edge }}$ (active sites) reduce with the increase in the growth time from $4.25 \%$ to approx. $1.45 \%$ for samples grown for 30 and $600 \mathrm{~s}$, respectively. These values of $\theta_{\text {edge }}$ are similar to those observed for graphite powder and carbon nanotubes $[5,6]$. It is also worth mentioning here that in the case of HOPG, the active sites are usually of the order of $0.5 \%$ only [6]. Hence, the FLGs show a much higher proportion of active edges as compared to HOPG and are comparable to the active sites on CNTs. As discussed before, the presence of defects can give rise to localised DOS. Since the DOS is dictated by the defect sites on edge planes, a structure with higher edges/defect sites would inevitably provide higher electron transfer rate. Consequently, the amount of edge planes observed reduces with the increase in the growth time via a reduction in the areal density of flakes, lowered defect density and associated DOS.

\section{Oxygen reduction reaction}

The cyclic voltammetry scans of pristine and N-doped FLGs in $\mathrm{O}_{2}$ saturated $0.1 \mathrm{M} \mathrm{KOH}$ electrolyte are shown in Fig. 5(a). It can be clearly observed that N-doped FLGs is electro-catalytically more active as compared to pristine FLGs as judged from the positive shift of the overpotential for ORR by nearly $40 \mathrm{mV}$ and the increase in the current density [14-18]. To obtain further insight into the electron transfer kinetics, the number of electrons $(n)$ participating in the reaction can be worked out using the current response at various scan rates (Fig. 5(b)) in conjunction with RandlesSevcik equation, given by:

$I_{p}=2.687 \times 10^{5} A C n^{\frac{3}{2}}(D v)^{\frac{1}{2}}$

where $I \mathrm{p}$ is the peak current in Amperes, $n$ is the number of electron participating in the reaction, $v$ is the scan rate in $\mathrm{V} / \mathrm{s}$, $D$ is the diffusion coefficient for oxygen in $0.1 \mathrm{M} \mathrm{KOH}$ $\left(1.73 \times 10^{-5} \mathrm{~cm}^{2} \mathrm{~s}^{-1}\right), A$ is the area of the electrode (in square centimetre) and $C$ represents the oxygen concentration in the electrolyte $\left(2.4 \times 10^{-7} \mathrm{molcm}^{-3}\right)[15,16,37]$. The calculated values show that ORR on pristine FLGs follows a twoelectron process $(n=2.3)$. For $300 \mathrm{~s}$ N-doped FLGs, the value of $n$ is 3.23; whereas for $600 \mathrm{~s} \mathrm{~N}$-doped FLGs, the value is 3.45 , suggesting a four-electron process for the ORR on the $\mathrm{N}$ doped FLGs. The results agree with previous reports where it has been shown that for N-doped FLGs, the ORR reaction usually proceeds through a four electron pathway, yielding water as the main end product $[15,21,37,38]$.

While the ability of $\mathrm{N}$-doped graphene to catalyse ORR has been reported widely, the ability of pristine graphene to catalyse ORR has been reported only recently $[18,21,37]$. The catalytic activity of the pristine FLGs can be explained by understanding the case of ORR on GCE via the formation of adsorbed superoxide ions on the glassy carbon surface [39, 40]. For $\mathrm{N}$-doped FLGs, the presence of nitrogen functional groups and its associated effects are responsible for the oxygen reduction [14-16]. In our studies, the maximum ORR response was observed from $600 \mathrm{~s}$ ECR N-doped FLGs (Fig. 5(a)). The high resolution XPS spectra of $\mathrm{C} 1 \mathrm{~s}$ and N1s regions of pristine and N-doped FLGs is
Fig. 5 Comparison of CV scans of a pristine FLGs and $300,600 \mathrm{~s}$ N-doped FLGs in $\mathrm{O}_{2}$ saturated $0.1 \mathrm{M} \mathrm{KOH}$ (taken at $50 \mathrm{mV} / \mathrm{s}$ ), b plot of peak current vs. square root of scan rate for pristine and N-doped FLGs, c comparison of cyclic voltammograms of $\mathrm{Pt} / \mathrm{CB}$ and pristine FLG in the absence and presence of $1 \mathrm{M} \mathrm{CH}_{3} \mathrm{OH}$ in $\mathrm{O}_{2}$ saturated $0.1 \mathrm{M} \mathrm{KOH}$ solution and $\mathbf{d}$ comparison of chronoamperometric scans of pristine FLGs and N-doped FLGs in $\mathrm{O}_{2}$ saturated $0.1 \mathrm{M}$ $\mathrm{KOH}$ solutions
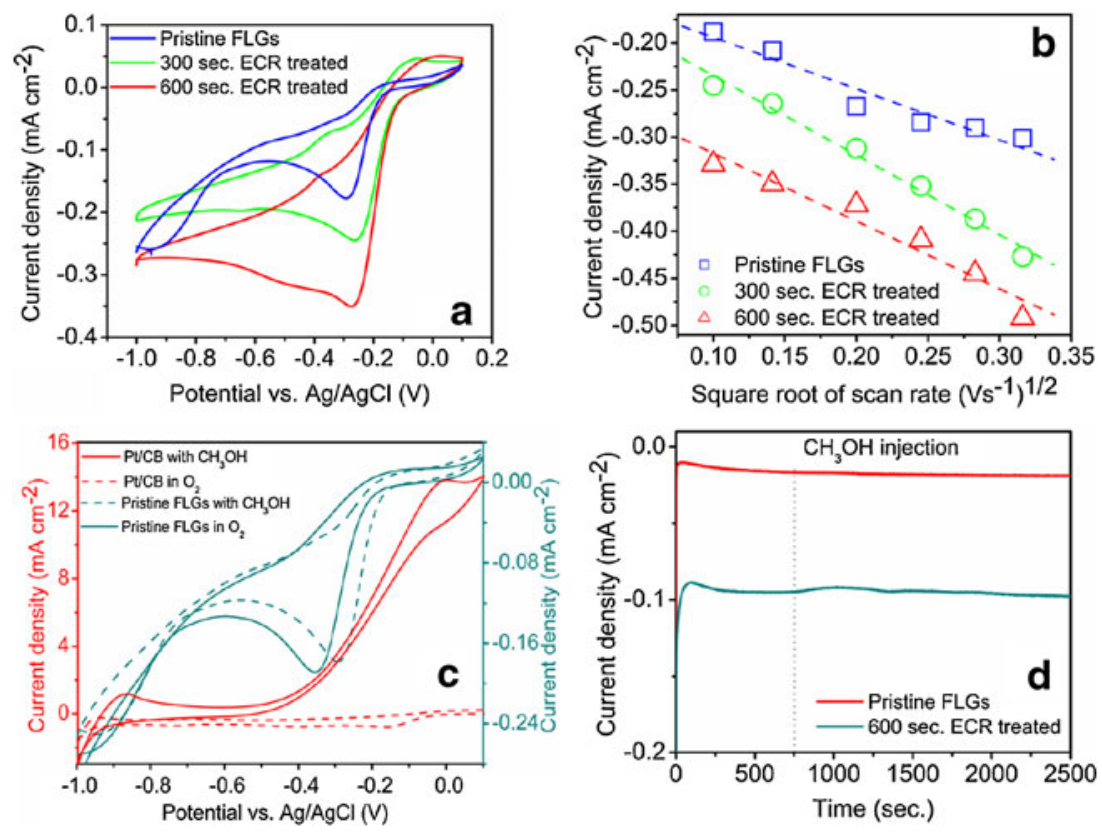
shown in Fig. 6 (a, b), while the surface elemental analysis is shown in Table 2. It can be clearly observed that the ECR plasma treatment was successful in modifying the surface of pristine FLGs by increasing the $\mathrm{N}$ content from $7.24 \%$ for $300 \mathrm{~s}$ treatment to $17.30 \%$ for $600 \mathrm{~s}$ treatment. The increase in the nitrogen content is also accompanied by an increase in the oxygen content due to the high reactivity of functionalised sites on the graphene flakes [10, 41]. It can be argued that with the increase in the $\mathrm{N}$ content, there is a corresponding increase in the defect density and oxygen content as well. Indeed, it has been shown in some recent reports that ORR can be observed in the case of O-doped graphene as well [18]. However, the response on O-doped ORR is not as strong as that observed on $\mathrm{N}$-doped graphene [18]. Also, since the reduction mechanisms for oxygen on $\mathrm{N}$ doped and O-doped electrodes are similar, it is difficult to distinguish between the two, unless individual moieties of nitrogen or oxygen can be grafted on the surface.
Hence, further discussion will be restricted to the effect of $\mathrm{N}$-doping by assuming that $\mathrm{O}$ moieties do not contribute significantly to the overall reduction current. Using the Randles-Sevcik equation, the effective electrode area is calculated to be $0.289 \mathrm{~cm}^{2}$ for pristine FLGs; whereas for ECR-treated FLGs, the effective electrode area is nearly $1.24 \mathrm{~cm}^{2}$ (see supporting information, S3). This huge increase in the effective area manifests itself into a large roughness factor of nearly 17.5 for ECR-treated FLGs as compared to 4.09 for pristine FLGs. The increase in the observed current density for N-doped FLGs can be explained in terms of the following: (1) increase in the effective electronic DOS near the Fermi level and (2) increase in the defect density leading to higher number of edge sites. This increase in the defect density can be thought as synonymous to an increase in the disorder of the material. This higher localised DOS caused by enhanced defect density could be responsible for increased current density while maintaining favourable fast electron
Fig. 6 High resolution XPS spectra of a $\mathrm{C} 1 \mathrm{~s}$ core region showing the broadening upon nitrogen doping, $\mathbf{b}$ increase in the N1s signal upon doping, $\mathbf{c}$ deconvolution of N1s spectra of N-doped FLGs (300 s), d deconvolution of N1s spectra of N-doped FLGs (600 s), e valence band spectra of pristine and $\mathrm{N}$-doped samples and $\mathbf{f}$ lowering of work function in $\mathrm{N}$ doped FLGs showing the enhancement of electronic DOS
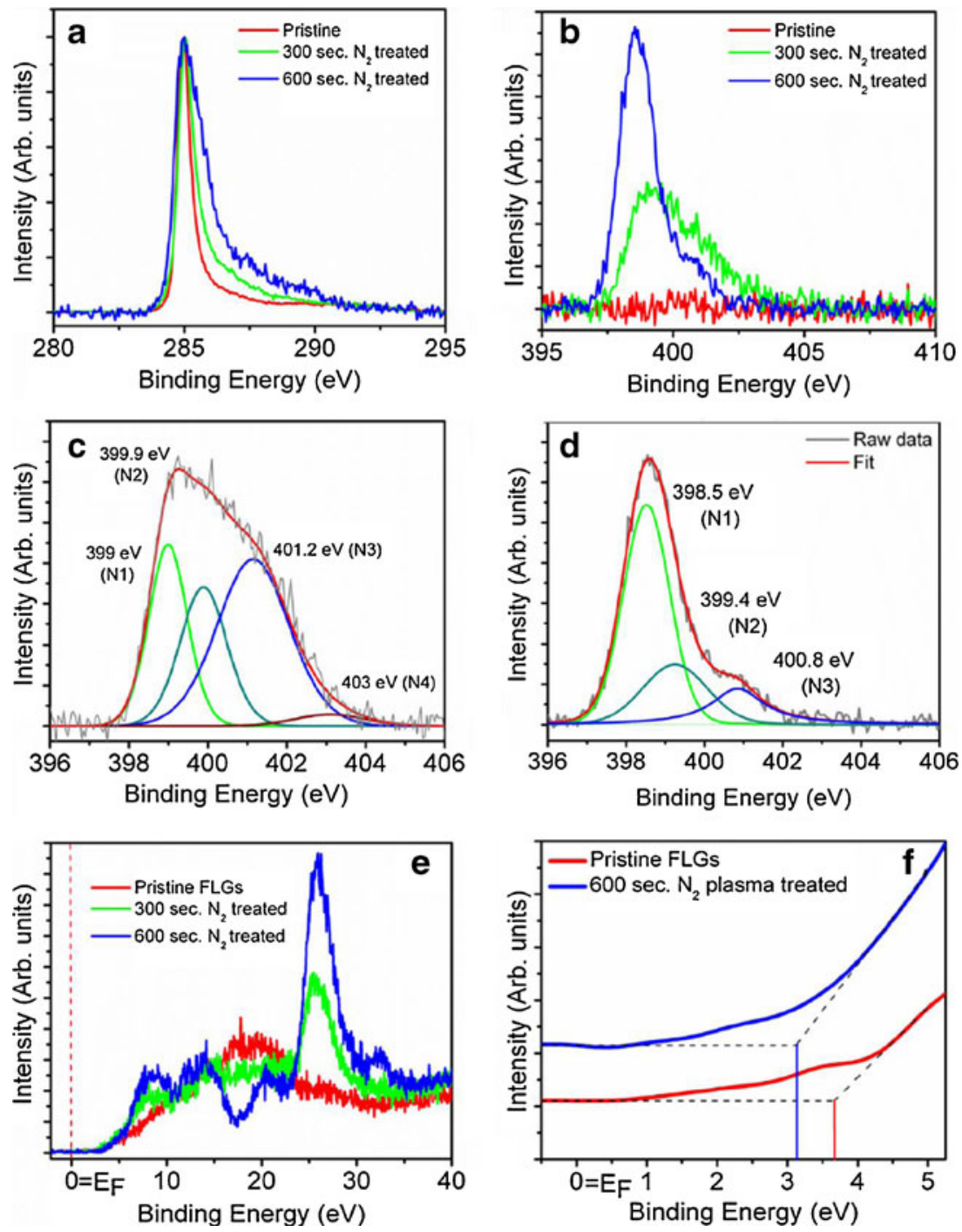
transfer kinetics on N-doped FLGs. Nevertheless, strong ORR has been recently reported in the case of nitrogendoped graphene quantum dots with oxygen rich functional groups, and it is believed that the presence of oxygen can further help in the creation of partial charge on attached carbon atom [14-16, 21]. From the deconvoluted N1s spectra that for $600 \mathrm{~s}$ sample, it can be observed that most of the nitrogen functionality is in the form of pyridinic sites only (peak N1), which can enhance the ORR activity (Fig. 6(c, d)). These results are in agreement with those observed by Kundu et al. [38] and Rao et al. [42], where increased ORR activity was ascribed to pyridinic groups. Using valence band XPS, we have previously shown that the effective electronic DOS near the Fermi level is enhanced upon $\mathrm{N}$ doping (Fig. 6 (e, f)) This enhanced DOS and enhanced electrochemical surface area can also contribute towards enhanced ORR (see supporting information S2, S3). As compared to carbon, both nitrogen and oxygen show higher-electronegativity with the order of electronegativity being $\mathrm{C}(2.55)<\mathrm{N}(3.04)<\mathrm{O}(3.44)$. Due to the electronegative nature of the both nitrogen and oxygen, any carbon atom to which they are attached will develop a partial positive charge [14-16]. Recent DFT calculations by Popov et al. have shown that the incorporated nitrogen itself is not the active site for ORR, but the carbon atom adjoining a nitrogen atom has a reduced energy barrier to ORR [43]. Also, the electron density of the carbon atom is reduced by the nitrogen doping making the adsorption of the oxygen molecule easier [44]. Moreover, nitrogen-doping is known to create surface defects leading to an increase in the edge plane exposure $[10,45]$. Recently, Ozkan et al. showed that for enhanced ORR, increased edge plane are needed in conjunction with nitrogen doping for high ORR activities [45]. Previous reports in the literature have suggested that pyridinic type nitrogen functionalities can provide enhanced ORR activity [15-18, 38, 42]. This has been explained using the conjugation effect between the lone pair of electrons of nitrogen and the $\pi$ electrons of graphene [14, 38, 42]. Stevenson et al. have reported on the electronic and electrochemical properties of carbon nanotubes [46]. In their studies, it was observed that $\mathrm{N}$ doping induces enhanced bulk electrical conductivity and chemically active sites with localised enhanced electron density [46]. These sites with enhanced electronic density may arise due to the presence of electron rich pyridinic site with a lone pair of electrons which work in conjunction with the conjugated $\pi$ electrons of graphene [46]. In our case, increased edge planes are observed via the creation of additional defect sites by the insertion of nitrogen in the carbon lattice. This insertion of nitrogen also enhances the localised DOS to provide enhanced ORR. In fact, by the use of
N-doped FLGs, non-enzymatic detection of hydrogen peroxide can be carried with enhanced sensitivity as compared to pristine FLGs (see supporting information, S3).

One of the major drawbacks of using transition metal catalyst such as Pt for ORR is that they are highly susceptible to methanol crossover effects [15]. In alcohol based fuel cells, the crossover of fuel from anodic to cathodic side could lower the performance through the depolarizing effect [15]. This depolarizing effect is observed as the oxidation of alcohols such as $\mathrm{CH}_{3} \mathrm{OH}$, which can commence at a lower potential than the ORR, thus producing poisonous species such as CO which can adversely affect the operation of the fuel cell system [14-18]. The response of $\mathrm{Pt} / \mathrm{CB}$ and pristine FLGs in $\mathrm{O}_{2}$ saturated solution, with and without the presence of $2 \mathrm{M} \mathrm{CH}_{3} \mathrm{OH}$ is shown in Fig. 5(c). The $\mathrm{Pt} / \mathrm{CB}$ electrode shows a much higher preference for $\mathrm{CH}_{3} \mathrm{OH}$ oxidation leading to the complete disappearance of ORR peak. In comparison, the ORR response from pristine FLGs remains stable. In fact, the response of pristine FLGs actually increases upon the addition of $\mathrm{CH}_{3} \mathrm{OH}$ which can be attributed to the increase in the oxygen species when $\mathrm{CH}_{3} \mathrm{OH}$ was added to the solution. In order to study the long-term stability of the samples, chronoamperometric studies were performed in $\mathrm{O}_{2}$ saturated $0.1 \mathrm{M} \mathrm{KOH}$ solutions for duration of 2,500 s (Fig. 5(d)). The samples were studied at their respective onset potentials, and hence the observed current is less than peak current density. For all the samples, $2 \mathrm{M}$ $\mathrm{CH}_{3} \mathrm{OH}$ was injected after $750 \mathrm{~s}$. Both the samples showed very stable response with the pristine FLGs retaining nearly $72 \%$ of its starting current, while the N-doped samples retained nearly $95 \%$ of its starting current. Thus, both pristine and N-doped FLGs show excellent and long-term electrochemical activity for ORR which is unhindered by the presence of methanol.

\section{Conclusion}

The FLG nanoflake films, as advanced electrode materials, were prepared and extensively investigated using a variety of electrochemical probes. The FLGs exhibit fast electron transfer kinetics which can be attributed to the high degree of edge planes, acting as the active sites for electrochemical reactions. An estimation of the edge plane active sites was carried out using a quantitative relationship between heterogeneous electron transfer rate and edge density. These studies aided in the understanding of the relationship between the microstructure, areal density and electrochemical properties of FLG electrodes. Furthermore, a facile plasma-based route for nitrogen doping of FLGs was developed. The N-doped FLGs show excellent performance for ORR in alkaline conditions with long-term stability and tolerance to methanol 
crossover. To conclude, both the pristine and N-doped FLGs provide an excellent platform to study the electrochemical behaviour of edge planes whilst providing further opportunities to create the next generation of functional electrodes for electrochemical applications.

\section{References}

1. McCreery RL (2008) Chem Rev 108:2646-2687

2. Rice RJ, McCreery RL (1989) Anal Chem 61:1637-1641

3. Dumitrescu I, Unwin PR, Macpherson JV (2009) Chem Commun 45:6886-6901

4. Brownson DAC, Banks CE (2010) Analyst 135:2768-2778

5. Davies TJ, Hyde ME, Compton RG (2005) Angew Chem 117:5251-5256

6. Hallam PM, Banks CE (2011) Electrochem Commun 13:8-11

7. Banks CE, Crossley A, Salter C, Wilkins SJ, Compton RG (2006) Angew Chem 45:2533-2537

8. Šljukic B, Banks CE, Richard G (2006) Nano Lett 6:1556-1558

9. Soin N, Roy SS, O'Kane C, Lim TH, Hetherington CJD, McLaughlin JAD (2011) CrystEnggComm 13:312-318

10. Soin N, Roy SS, Roy S, Hazra KS, Misra DS, Lim TH, Hetherington CJD, McLaughlin JAD (2011) J Phys Chem C115:5366-5372

11. Soin N, Roy SS, Lim TH, McLaughlin JAD (2011) Mater Chem Phys 129(3):1051-1057

12. Soin N, Roy SS, Mitra SK, Thundat SK, McLaughlin JAD (2012) J Mater Chem 22:14944-14950

13. Pumera M, Sasaki T, Iwai H (2008) Chem Asian J 3:2046-2055

14. Yu D, Nagelli E, Du F, Dai L (2010) J Phys Chem Lett 1:2165-2173

15. Gong K, Du F, Xia Z, Durstock M, Dai L (2009) Science 323:760

16. Sheng ZH, Shao L, Chen JJ, Bao WJ, Wang FB, Xia XH (2011) ACS Nano 5:4350-4358

17. Qu L, Liu Y, Baek JB, Dai L (2010) ACS Nano 4:1321-1326

18. Shao Y, Zhang S, Engelhard MH, Li G, Shao G, Wang Y, Liu J, Aksay IA, Lin Y (2010) J Mater Chem 20:7491-7496

19. Shao Y, Sui J, Yin G, Gao Y (2008) Appl Catal, B 79:89-99

20. Liu R, Wu D, Feng X, Müllen K (2010) Angew Chem 122:2619-2623

21. Li Y, Zhao Y, Cheng H, Hu Y, Shi G, Dai L, Qu L (2012) J Am Chem Soc 134:15-18

22. Lee YH, Lee JH (2009) Appl Phys Lett 95:143102
23. Shang NG, Papakonstantinou P, McMullan M, Chu M, Stamboulis A, Potenza A, Dhesi SS, Marchetto H (2008) Adv Funct Mater 18:3506-3514

24. Jones CP, Jurkschat K, Crossley A, Richard G, Riehl BL, Banks CE (2007) Langmuir 23:9501-9504

25. Tang L, Wang Y, Li Y, Feng H, Lu J, Li J (2009) Adv Funct Mater 19:2782-2789

26. Lin YG, Hsu YK, Wu CT, Chen SY, Chen KH, Chen LC (2009) Diamond Relat Mater 18:433-437

27. Zoski CG (2007) Handbook of electrochemistry. Elsevier, Netherlands

28. Bard AJ, Faulkner LR (2001) Electrochemical MethodsFundamentals and applications. John Wiley \& Sons

29. Hrapovic S, Liu YL, Male KB, Luong JHT (2004) Anal Chem 76:1083-1088

30. Salinas-Torres D, Huerta F, Montilla F, Morallón E (2011) Electrochim Acta 56:2464-2470

31. Pacios M, Valle M, Bartroli J, Esplandiu MJ (2008) J Electroanal Chem 619:117-124

32. Nicholson RS (1965) Anal Chem 37:1351-1355

33. Luais E, Boujtita M, Gohier A, Tailleur A, Casimirius S, Djouadi MA, Granier A, Tessier PY (2010) Appl Phys Lett 96:126103

34. Davies TJ, Banks CE, Compton RG (2005) J Solid State Electrochem 9:797-808

35. Kobayashi K (1993) Phys Rev B 48:1757

36. Ji X, Banks CE, Crossley A, Compton RG (2006) ChemPhysChem 7:1337-1344

37. Yang SY, Chang KH, Huang YL, Lee YF, Tien HW, Li SM, Lee YH, Liu CH, Ma CM, Hu CC (2012) Electrochem Commun 14:39-42

38. Kundu S, Nagaiah TC, Xia W, Wang Y, Dommele SV, Bitter JH, Santa M, Grundmeier G, Bron M, Schuhmann W (2009) J Phys Chem C 113:14302-14310

39. Yeager E (1986) J Mol Catal 38:5-25

40. Paliteiro C, Hamnett A, Goodenough JB (1987) J Electroanal Chem 233:147-159

41. Abbas G, Papakonstantinou P, Iyer GRS, Kirkman IW, Chen CL (2007) Phys Rev B 75:195429

42. Rao CV, Cabrera CR, Ishikawa Y (2010) J Phys Chem Lett 1:26222627

43. Sidik RA, Anderson AB, Subramanian NP, Kumaraguru SP, Popov BN (2006) J Phys Chem B 110:1787-1793

44. Okamoto Y (2009) Appl Surf Sci 256:335-341

45. Matter PH, Zhang L, Ozkan US (2006) J Catal 239:83-96

46. Wiggins-Camacho JD, Stevenson KJ (2009) J Phys Chem C 113:19082-19090 